\title{
Superimposition of Multiple Leads
}

National Cancer Institute

\section{Source}

National Cancer Institute. Superimposition of Multiple Leads. NCI Thesaurus. Code C117760.

Measurement methodology using multiple leads for the determination of an ECG parameter. This is often visually represented as a superimposition of the median beats from multiple leads. (CDISC) 